\title{
The Informative Value of Several Clinical-laboratory Characteristics at the Time of Hospitalization in Terms of Prognosis of Disease Complication with COVID-19 Patients
}

\section{Ratiani ${ }^{1,2}$, T Sanikidze ${ }^{2}$, G Ormotsadze ${ }^{2,3}$, E Vashakidze $^{1,2}$, V Shoshiashvili1 ${ }^{1,2 *}$, E Pachkoria ${ }^{1,2}$, M Rurua ${ }^{1,2}$, E Khuchua ${ }^{1,2}$, L Gabunia $^{1,2}$, K Machavariani ${ }^{1,2}$ and T Megrelishvilii ${ }^{1,2}$}

${ }^{1}$ First University Clinic of Tbilisi State Medical University, Georgia

${ }^{2}$ Tbilisi State Medical University, Georgia

${ }^{3}$ I. Beritashvili Center of Experimental Biomedicine, Georgia

*Corresponding Author: V Shoshiashvili, First University Clinic of Tbilisi State

Medical University, Georgia.
Received: July 16, 2021

Published: August 28, 2021

(C) All rights are reserved by $\mathbf{V}$ Shoshiashvili., et al.

\section{Abstract}

The number of patients with COVID-19 is currently rapidly increasing globally. Most of the patients with COVID-19 have a favorable prognosis, but some have rapidly progressed to severe and critical conditions with respiratory distress syndrome, coagulation dysfunction, multiple organ failure, etc.

The purpose of our study was to identify early clinical-laboratory indicators of possible complications in COVID-19 patients.

The information of a total of 106 patients with confirmed COVID-19 (the positive result of the nucleic acid test by real-time fluorescence RT-PCR), who were admitted to First University Clinic of Tbilisi State Medical University (Georgia) (1.04.20-1.5.20), was collected. The clinical-laboratory data (saturation index, $\mathrm{pH}$, hemoglobin, leukocytes (lymphocytes and neutrophils) and thrombocytes content, prothrombin, D-Dimer, CRP, LDH, AST, ALT, bilirubin, urea level in the blood, and CT-rate) were collected at the time of patient admission to the hospital.

In accordance with the severity of the course of the disease during hospitalization, all patients were divided into two groups without complications and with severe complications, when respiratory and other organs failure occur, requiring observation and treatment in intensive care units (ICU).

Exploratory and confirmatory (K-mean Clustering, ANOVA, Xi2), data analysis methods were used to reveal the statistical reliability of the difference between the mean values of the parameters. To identify the optimal complex predictors of the risk of complications, Binary Logistic Regression analysis and Receiver operating characteristic (ROC) curve analysis were performed.

According to the results of the study, it was found that the prognostic criterion of further complications in patients with COVID-19, developed on the basis of a linear function of parameter values (saturation index, neutrophil count, and CT-Rate) at the moment of hospitalization is characterized by high accuracy. Also, the ways for further refining the complex prognostic criterion were identified. In particular, the use of generalized nonlinear models with random effects in regression will allow to 1) increase the number of clinical- laboratory characteristics, 2) consider the effects of interaction between them, 3) to assess the level of uncertainty and its sources.

Citation: V Shoshiashvili., et al. "The Informative Value of Several Clinical-laboratory Characteristics at the Time of Hospitalization in Terms of Prognosis of Disease Complication with COVID-19 Patients". Acta Scientific Medical Sciences 5.9 (2021): 143-149. 
The Informative Value of Several Clinical-laboratory Characteristics at the Time of Hospitalization in Terms of Prognosis of Disease Complication with COVID-19 Patients

The studied cohort was quite limited (106 patients) and given the importance of comorbidities in the development of complications of COVID-19, it is unlikely to be representative of the general population. It is important to increase the studied cohort's capacity, which will allow us to take into account the effects of comorbidity, the degree of representativeness, and perform a comparative analysis of various therapeutic approaches, generalize the results to other populations.

Keywords: COVID -19; Clinical-laboratory; Indicators; Complication; Predictor

\section{Introduction}

The number of patients with COVID-19 is currently rapidly increasing globally [1]. Most of the patients with COVID-19 have a favorable prognosis, but some have rapidly progressed to severe and critical complications with respiratory distress syndrome, coagulation dysfunction, multiple organ failure, etc. [2,3].

The COVID-19 pandemic has a high impact on public health in many countries [4]. Except for a few countries that have succeeded in early control of the epidemic's transmission chains (South Korea, Hong Kong) [5,6], most countries (China, Italy, France, Spain, UK, USA, and Brazil) have experienced consequences of rapidly spreading epidemic pattern, were not prepared enough for its growing scale and especially for the flow of critically ill patients requiring intensive care units (ICU).

The main targets of pathological changes during COVID-19 are the lung (serous, fibrin exudate, and clear membrane in the alveolar cavity, and congestion and edema in the lung [7]) and immune system [8]. CT scan examination is a quick and simple method to screen the characteristic imaging of lung changes and can often predict the prognosis of patients with COVID-19. The sensitivity of COVID-19 diagnosis with CT scan alone was 76.4\%, therefore, the application of CT scan in COVID-19 was evaluated as useful [9].

Early monitoring of sensitive clinical-laboratory indicators of patients with COVID- 19, good correlated with manifested by CT -manifested pathological alterations in the lungsin the early stages of the disease, can be used to identify markers of possible complications and severity of the disease at its more later stages.

This can be useful for both public health policy development and hospital management, providing an important framework for guiding treatment strategies and preventing complications and patient deaths.

\section{Purpose of the Study}

The purpose of our study was to identify early clinical-laboratory indicators of possible complications in COVID-19 patients.

\section{Materials and Methods}

\section{Source of patients and diagnosis criteria}

The information of a total of 106 patients with confirmed COVID-19 who were admitted to First University Clinic of Tbilisi State Medical University (Georgia) (April 1, 2020 May 1, 2020) was collected. The confirmed patients had a positive result of the nucleic acid test of COVID-19 by real-time fluorescence RT-PCR.

\section{Data collection}

The clinical-laboratory data (saturation index, $\mathrm{pH}$, hemoglobin, leukocytes (lymphocytes and neutrophils) and thrombocytes content, prothrombin, D-Dimer, CRP, LDH, AST, ALT, bilirubin, urea level in the blood, and CH-rate) were collected at the time of patients' admission to the hospital.

Clinical assessment of disease severity was conducted using clinical symptoms and CT scan results. In the first stage, patients were divided into four groups according to the severity of the disease at the time of hospitalization (mild cases (1), middle cases (2), severe cases (3), and critical cases (4)). Mild cases (1) had mild clinical symptoms and no pneumonia manifestation in CT imaging (CT< 3 scores). Moderate cases (2) had fever and respiratory tract symptoms, and pneumonia manifestation can be seen (CT $<8$ scores). Severe cases (3) met any of the following: respiratory distress, RR $\geq 30$ breaths/min, the oxygen saturation less than $93 \%$ at a rest state ( $\mathrm{CT}<16$ scores). Patients with $>50 \%$ lesion progress within 24 to 48 hours in pulmonary imaging were treated as critical cases (4).

In accordance with the severity of the course of the disease during hospitalization, all patients were divided into two groups 
- without complications (group I) and with severe complications (group II), when a respiratory failure occurs, and mechanical ventilation is required, or complications of other organs requiring monitoring and treatment in intensive care units (ICU).

The retrospective case series study was performed; no patients were involved in the plan design, conducting the research procedures or the measurements directly. No patients were asked the written consent on participation in the study and interpretation of the results.

\section{Statistical methods}

Exploratory and confirmatory (K-mean Clustering, ANOVA, $\mathrm{Xi}^{2}$ ) data analysis methods were used to reveal the statistical reliability of the difference between the mean values of the parameters.

To identify the optimal complex predictors of the risk of complications, Binary Logistic Regression analysis and Receiver operating characteristic (ROC) curve analysis [10] was performed.

The receiver operating characteristic (ROC) curve and area under the ROC curve (AUC) were used to analyze the optimal cut-off for the prediction of positive RT-PCR cases. In this study, AUC $=0.9$ to 1 was defined as excellent accuracy, 0.8 to 0.9 as very good, and AUC $<0.8$ as poor (useless test).

Logistic regression was used to identify the causal relationship between the values of the selected characteristics and the likelihood of disease severity:

$$
P(+)=\frac{E X P(Z)}{1+E X P(Z)}
$$

Where $\mathrm{P}(+)$ is the probability that a particular patient with specific characteristics falls into the category of severe patients. Only linear combinations of individual parameters were considered at this stage:

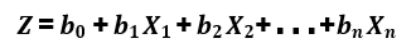

Where $b_{i}$ - regression coefficients; $\left\{\boldsymbol{X}_{i}\right\}_{i=1}^{n}-n$ values of $\mathrm{i}$ indicators of rescaled and re-centered to the physiological norm.
$X_{i}=\frac{X^{\prime}=\bar{x}}{\operatorname{STD}\left(X^{\prime}\right)}$

For primary processing, analysis, and graphical visualization of data, we used MATLAB R-2020 and STATISTICA-12 mathematical software packages.

\section{Results}

\section{Characteristics of the studied cases}

Among 106 patients with COVID-19, the average age was 49.02 \pm 1.97 (Min_Max $\div 11-86$ ) years, over 60 years there were $30 \%$ of cases; men accounted for $42.5 \%$ of cases, women - $57.5 \%$.

\section{Laboratory parameters}

Table 1 shows Mean values, Confidence Interval, Fisher F criterium, and Statistical Significance of Difference in clinical-laboratory characteristics (saturation index, $\mathrm{pH}$, hemoglobin, leukocytes (lymphocytes and neutrophils) and thrombocytes content, prothrombin, D-Dimer, CRP, LDH, AST, ALT, bilirubin, urea level in blood and CT-rate) of patients with an uncomplicated (group I) and severe-complicated (group II) course of COVID- 19 during hospitalizing period.

Figure 1 shows the dependence of the mean values, Standard Error end Confidence Interval in a number of clinical and laboratory parameters on the severity of the patient's state at the time of hospitalization in patients with uncomplicated (group I) and severe-complicated (group II) course of COVID-19.

Regression coefficients, Standard Error, and p-value of multivariable linear discriminant function were calculated for predicting the high-risk patients' group (group II) (Logistic Regression, Binomial Distribution). Table 2 presents the results of Logistic Regression Analysis specifically for parameters with high statistical significance of the difference between groups I and II.

Table 3 and figure 2 is shown an ensemble of optimal characteristics (Saturation, Neutrophils, CT-Rate) and a discriminant function based on them, which with acceptable efficiency (AUC $=0.96$ ) distinguishes groups of patients with and without complications.

Figure 3 presents the values of the discriminant function $(\boldsymbol{Z})$ in uncomplicated (0) and complicated (1) patients. 
The Informative Value of Several Clinical-laboratory Characteristics at the Time of Hospitalization in Terms of Prognosis of Disease Complication with COVID-19 Patients

\begin{tabular}{|c|c|c|c|c|}
\hline \multirow{2}{*}{ Characteristics } & Group I & Group II & \multirow{2}{*}{ Fisher F } & \multirow{2}{*}{$\mathbf{p}$} \\
\hline & Mean; \pm 95\%CI & Mean; \pm 95\%CI & & \\
\hline Age & $44.66 ;(40.65 \div 48.68)$ & $65.15 ;(56,56 \div 73.74)$ & 18.37 & $>0.001$ \\
\hline Saturation & $95.94 ;(95.33 \div 96.55)$ & $90.20 ;(88.92 \div 91.47)$ & 64.87 & $>0.001$ \\
\hline $\mathrm{Ph}$ & $95.94 ;(95.33 \div 96.55)$ & $7.32 ;(7.29 \div 7.35)$ & 8.67 & 0.003 \\
\hline $\mathrm{Hb}$ & $13.60 ;(13.22 \div 13.98)$ & $12.55 ;(11.73 \div 13.36)$ & 5.41 & 0.022 \\
\hline Leukocyte & $6.31 ;(5.76 \div 6.86)$ & $7.59 ;(6.44 \div 8.74)$ & 3.93 & 0.050 \\
\hline Limphocytes & $1.67 ;(1.53 \div 1.89)$ & $1.17 ;(0.89 \div 1.46)$ & 9.75 & 0.002 \\
\hline Neutrophils & $0.60 ;(0.55 \div 0.62)$ & $0.77 ;(0.72 \div 0.82)$ & 34.69 & $>0.001$ \\
\hline D-Dimer & $431 ;(322 \div 541)$ & 1038; $(811 \div 1264)$ & 22.99 & $>0.001$ \\
\hline Thrombocytes & $246 ;(232 \div 261)$ & $220 ;(189 \div 252)$ & 2.17 & 0.14 \\
\hline Prothrombin & $15.00 ;(14.50 \div 15.50)$ & $15.33 ;(14.28 \div 16.33)$ & 0.37 & 0.54 \\
\hline Bilirubin & 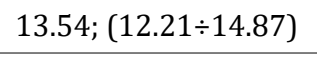 & 14.77; $(11.89 \div 17.57)$ & 0.56 & 0.45 \\
\hline CRP & $14.10 ;(1.41 \div 26.79)$ & $99.83 ;(73 \div 126)$ & 34.10 & $>0.001$ \\
\hline LDH & $200 ;(177 \div 222)$ & $447 ;(395 \div 500)$ & 74.73 & $>0.001$ \\
\hline AST & $21.32 ;(19.36 \div 23.27)$ & $37.06 ;(32.52 \div 41.60)$ & 40.15 & $>0.001$ \\
\hline ALT & $25.89 ;(20.56 \div 31.22)$ & $30.77 ;(18.40 \div 43.14)$ & 0.51 & 0.47 \\
\hline Urea & $4.71 ;(3.83 \div 5.58)$ & $9.38 ;(7.53 \div 11.25)$ & 20.15 & $>0.001$ \\
\hline CT-Rate & $2.85 ;(1.92 \div 3.78)$ & $12.16 ;(10.24 \div 14.08)$ & 75.09 & $>0.001$ \\
\hline
\end{tabular}

Table 1: Mean values, Confidence Interval, Fisher F criterium, and Statistical Significance of Difference in clinical-laboratory characteristics of patients with an uncomplicated (group I) and severe-complicated (group II) course of COVID-19 during the hospitalizing period.

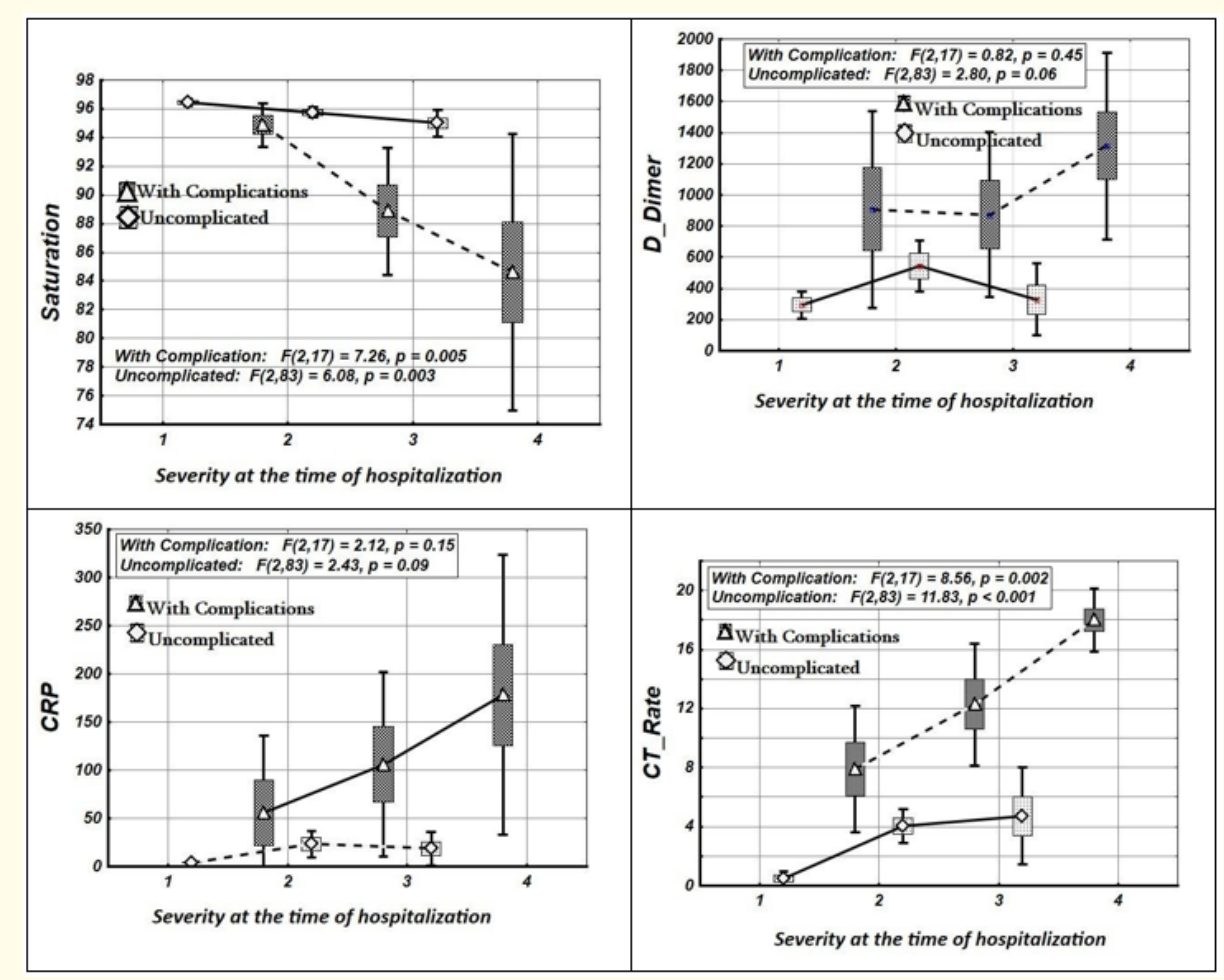

Figure 1: Dependence of the Values, Confidence Interval, and Statistical Significance of Differences of saturation index, D-Dimer, CRP content, and CT-Rate values on the severity of the patient's state at the time of hospitalization in patients with uncomplicated (group I) and severe-complicated (group II) course of COVID-19 (dark and light, respectively, groups of patients with and without complications during hospitalization period). 
The Informative Value of Several Clinical-laboratory Characteristics at the Time of Hospitalization in Terms of Prognosis of Disease Complication with COVID-19 Patients

\begin{tabular}{|l|l|l|l|}
\hline \multicolumn{3}{|l|}{ Logistic Regression, Binomial Distribution } \\
\hline $\begin{array}{l}\text { Dependent } \\
\text { Variables }\end{array}$ & b & Standard Error & P \\
\hline Age & 0.0725 & 0.04513 & 0.166 \\
\hline Saturation & $-0.42674^{*}$ & $0.18322^{*}$ & $0.019^{*}$ \\
\hline Neutrophils & 11.139 & 6.1204 & $0.062^{*}$ \\
\hline AST & $0.11832^{*}$ & $0.057172^{*}$ & $0.038495^{*}$ \\
\hline D-Dimer & -0.001 & 0.00093 & 0.110 \\
\hline CT Rate & 0.089 & 0.124 & 0.472 \\
\hline
\end{tabular}

Table 2: Regression coefficient, Standard Error, and p-value of multivariable linear discriminant function for predicting the difference between groups I and II.

Statistically significant changes.

\begin{tabular}{|l|l|l|l|}
\hline \multicolumn{4}{|l}{ Logistic Regresion, Binomial Distribution } \\
\hline $\begin{array}{l}\text { Dependent } \\
\text { Variables }\end{array}$ & Estimation (E) & Standard Error & P \\
\hline Saturation & -0.517 & 0.307 & 0.027 \\
\hline Neutrophils & 20.896 & 8.379 & 0.026 \\
\hline CT-Rate & 0.3 & 0.08 & 0.060 \\
\hline
\end{tabular}

Table 3: Regression coefficients, Standard Error, and p-value of multivariable linear discriminant function for predicting the difference between groups I and II.

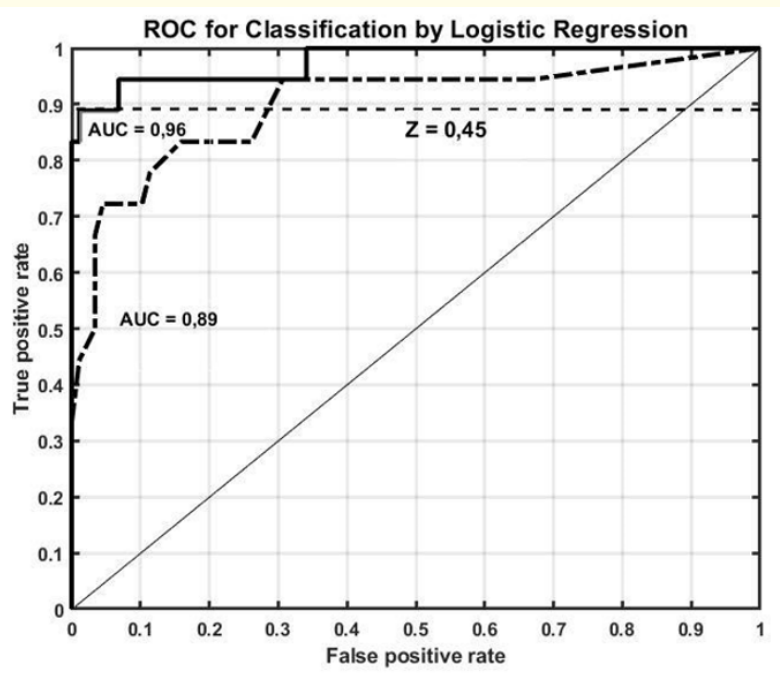

Figure 2: The clinical efficacy of the selected complex criterion evaluated according to the AUC value.

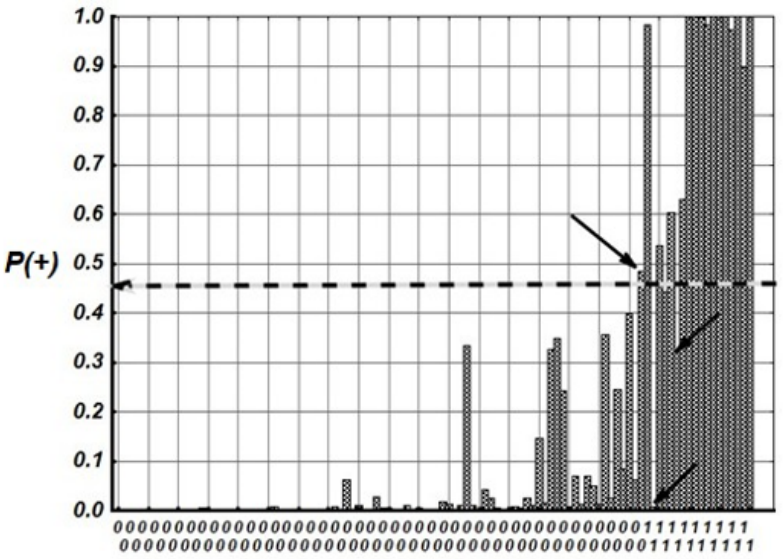

Figure 3: The values of the discriminant function $(\mathrm{Z})$ in uncomplicated (0) and complicated (1) patients (arrows indicate incorrectly identified cases).

\section{Discussion}

According to the data, that are shown in table 1, in patients groups with severe complications (transferred to the ICU) and uncomplicated course of COVID-19 during the period of hospitalization, a statistically significant difference was found between the values of some of the clinical-laboratory parameters (age, blood oxygen saturation, $\mathrm{pH}$, leukocytes (lymphocytes and neutrophils), D-dimer, CRP, HDL, AST, urea content, and CT-rate). It should be noted that given the high lethality during COVID-19 complication and the particular specificity of each case, even the high reliability of intergroup differences between characteristics may not be a sufficient condition for their clinical use.

We performed an analysis of Values, Confidentiality Interval, and Statistical Reliability of the Difference of a number of clinicallaboratory parameters in patients with uncomplicated (group I) and severe complicated (group II) course of COVID-19 during the hospitalization period, taking into account their severity at the time of the hospitalization. Figure 1 clearly shows that in patients of groups I and II the Values of Confidence Interval, and Statistical Significance of Differences in a number of clinical and laboratory parameters depend on the severity of the patient's state at the time of hospitalization. As evident from figure 1, except for CT-Rate, the coincidence of Confidence Intervals of characteristic values and, in some cases, the ranges of their Mean Standard Errors are recorded, indicating the low clinical efficacy of the criteria. 
As is known from the literature, both the risk of complications and its outcome are significantly dependent on factors such as the spectrum of concomitant chronic diseases (coronary artery disease, diabetes, hypertension, chronic obstructive pulmonary syndrome, etc.), the tactics and strategy of therapeutic procedures in the post-hospitalization period, population-specificity concerning treatment, etc. [11-13], which in the case of limited study cohorts are an additional source of uncertainty. Therefore, we considered it expedient to search for prognostic criteria based on a set of clinicallaboratory characteristics (complex characteristics), and not based on any single one. At a given stage of the study, complex criteria were searched in the form of linear combinations of clinical-laboratory characteristics (Table 2).

Table 2 presents the results of Logistic Regression Analysis specifically for parameters with high statistical significance of the difference between groups I and II. As it can be seen from Table 2, such highly informative characteristics from table 1, as CT- rate, D-dimer, age do not make a statistically significant contribution to discriminant plane regression. Estimation of the regression coefficients $(b)$ of the clinical-laboratory characteristics, which characterize the weight of a particular characteristic in the discriminant function (predictive value), their values, and the statistical reliability reflect the role of the corresponding factor in the development of the complication.

As shown in table 2, such highly informative characteristics as CT-Rate, D-Dimer, Neutrophils, do not make a statistically significant contribution to the discriminant plane.

This fact is related to the causal relationship between these characteristics, which can not be described by the linear function, which reduces their prognostic informativeness under linear approximation conditions and therefore limits the number and the type of parameters to be considered in the discriminant function. We selected an ensemble of optimal characteristics (Saturation, Neutrophils, CT-Rate) and a discriminant function based on them (Table 3), which with acceptable efficiency (AUC $=0.96$ ) distinguishes groups of patients with and without complications (Figure 2 ). Figure 2 shows the clinical efficacy of the selected complex criterion, which is evaluated according to the AUC value. For complex parameters, AUC $=0.96$, while for individual parameters it does not exceed 0,9 .
To evaluate the optimal value of sensitivity and specificity of criterium of possible complication of course of COVID-19 in patients during hospitalization, let consider figure 3 , which presents the values of the discriminant function $(\mathrm{Z})$ in uncomplicated (0) and sever- complicated (1) patients. As it turns out, the acceptable accuracy of discrimination of complicated and uncomplicated patients is achieved for a value that ensures the sensitivity of the criterion $=0.89(89 \%$ of complicated patients are correctly identified) and specificity $=0.02$ ( $92 \%$ of non-complicated patients are correctly identified).

\section{Limitation}

It should be noted, that the studied cohort was quite limited (106 patients), and therefore, given the importance of comorbidities in the development of complications of COVID-19, it is unlikely to be representative of the general population. In this regard, it is considered less appropriate the generalization of the complex criterion parameterized according to the examined cohort, due to its probable low representativeness, however, the clinical efficacy of using the complex criterion is obvious in the present article.

In future investigations it is important to increase the studied cohort's capacity, which will allow us to take into account the effects of comorbidity, the degree of representativeness and comparative analysis of various therapeutic approaches, its generalization to other populations.

\section{Conclusion}

According to the results of the study, it was found that the prognostic criterion of further complications in patients with COVID-19, developed on the basis of a linear function of parameter values (saturation index, neutrophil count, and CT-Rate) at the moment of hospitalization is characterized by high accuracy. Several parameters have a cyclical character, which probably reflects the body's adaptive response to infection and therefore introduces a large dispersion in the results.

The ways for further refining the complex prognostic criterion were identified. In particular, the use of generalized nonlinear models with random effects in regression allows us to 1) increase the number of clinical-laboratory characteristics, 2) consider the effects of interaction between them, 3) assess the level of uncertainty and its sources. 


\section{Bibliography}

1. Machase E. "China coronavirus: mild but infectious cases may make it hard to control outbreaks. Report warns". BMJ 368 (2020): m325.

2. W Han., et al. "The course of clinical diagnosis and treatment of a case infected with coronavirus disease 2019". Journal of Medical Virology 92.5 (2020): 461-463.

3. N Tang., et al. "Abnormal coagulation parameters are associated with poor prognosis in patients with novel coronavirus pneumonia". Journal of Thrombosis and Haemostasis 18.4 (2020): 844-847.

4. Kandel N., et al. "Health security capacities in the context of COVID-19 outbreak: an analysis of International Health Regulations annual report data from 182 countries". Lancet 395.10229 (2020): 1047-1053.

5. Shim E., et al. "Transmission potential and severity of COVID-19 in South Korea". International Journal of Infectious Diseases 93 (2020): 339-344.

6. Cowling BJ., et al. "Impact assessment of non-pharmaceutical interventions against coronavirus disease 2019 and influenza in Hong Kong: an observational study". Lancet Public Health 5.5 (2020): e279-288.

7. Xu Z., et al. "Pathological findings of COVID-19 associated with acute respiratory distress syndrome". Lancet Respiratory Medicine 8.4 (2020): 420-422.

8. General office of the national health commission of China. "Covid-19 diagnostic and therapeutic regimen (trial 7th, edition)". Journal of Cardiopulmonary Rehabilitation and Prevention 39.2 (2020): 103-107.

9. Lee KS. "Pneumonia associated with 2019 novel coronavirus: can computer tomographic findings help predict the prognosis of the disease?" Korean Journal of Radiology 21.3 (2020): 257-258.

10. Jayawant N Mandrekar. "Receiver Operating Characteristic Curve in Diagnostic Test Assessment”. Thoracolumbar Oncology 5 (2010): 1315-1316.

11. Tomasz J Guzik., et al. "COVID-19 and the cardiovascular system: implications for risk assessment, diagnosis, and treatment options". Cardiovascular Research 116.10 (2020): 16661687
12. Arun Samidurai and Anindita Das. "Cardiovascular Complications Associated with COVID-19 and Potential Therapeutic Strategies". International Journal of Molecular Sciences 21 (2020): 6790.

13. You Shang., et al. "Management of critically ill patients with COVID-19 in ICU: statement from front-line intensive care experts in Wuhan, China". Annals of Intensive Care 10 (2020): 73.

\section{Volume 5 Issue 9 September 2021}

(C) All rights are reserved by $\mathrm{V}$ Shoshiashvili., et al. 